\title{
Estimación de la resistencia a la compresión del hormigón por medio del ensayo de Velocidad de Pulso Ultrasónico (VPU)
}

\author{
N. G. Cayo-Chileno ${ }^{1 *}$, C. Moran-Velásquez ${ }^{1}$, J. H. A. Rocha ${ }^{2}$ \\ *Autor de Contacto: nahum.cayo.chileno@gmail.com \\ ${ }^{1}$ Departamento de Ingeniería Civil, Facultad de Tecnología, Universidad Privada del Valle, Cochabamba, Bolivia \\ ${ }^{2}$ Programa de Engenharia Civil, Universidade Federal do Rio de Janeiro, Rio de Janeiro, Brasil
}

\begin{abstract}
RESUMEN
El presente estudio tiene por objetivo estimar la resistencia a la compresión del hormigón mediante el ensayo de Velocidad de Pulso Ultrasónico (VPU). Se elaboraron 6 prismas de hormigón $(20 \mathrm{~cm}$ x $20 \mathrm{~cm}$ x $25 \mathrm{~cm}$ ) para dos resistencias a la compresión de diseño (fc'), 21 y $28 \mathrm{MPa}$, totalizando 12. Se aplicó el ensayo de VPU por transmisión indirecta a diferentes distancias $(5 \mathrm{~cm}, 10 \mathrm{~cm} \mathrm{y}$ $15 \mathrm{~cm}$ ). Los resultados de VPU y de resistencia a la compresión de los prismas fueron analizados mediante regresiones lineales. Los modelos lineales de cada $\mathrm{fc}^{\prime}$ por separado no son estadísticamente significativos; no obstante, el modelo que considera todos los datos (ambos fc') presenta un mejor ajuste.
\end{abstract}

Palabras clave: Regresión lineal; Transmisión indirecta; Cuerpos prismáticos.

\begin{abstract}
The objective of this study is to estimate the compressive strength of concrete by Ultrasonic Pulse Velocity (UPV) test. 6 concrete prisms $(20 \mathrm{~cm} \times 20 \mathrm{~cm} \times 25 \mathrm{~cm})$ were made for two design compressive strengths (fc'), 21 and $28 \mathrm{MPa}$, totaling 12. UPV test was applied by indirect transmission at different distances $(5 \mathrm{~cm}, 10 \mathrm{~cm}$ and $15 \mathrm{~cm})$. UPV and compressive strength results of the prisms were analyzed using linear regressions. The linear models of each fc' separately are not statistically significant; however, the model that considers all the data (both fc') presents a better fit.
\end{abstract}

Keywords: Linear regression; Indirect transmission; Prismatic specimen.

\section{RESUMO}

O objetivo do presente estudo é estimar a resistência à compressão do concreto por meio do ensaio Velocidade de Pulso Ultrassônico (VPU). Foram confeccionados 6 prismas de concreto $(20 \mathrm{~cm} \mathrm{x}$ $20 \mathrm{~cm}$ x $25 \mathrm{~cm}$ ) para duas resistências à compressão de projeto (fc'), 21 e $28 \mathrm{MPa}$, totalizando 12 . O ensaio VPU foi aplicado por transmissão indireta em diferentes distâncias $(5 \mathrm{~cm}, 10 \mathrm{~cm}$ e 15 $\mathrm{cm})$. Os resultados de VPU e resistência à compressão dos prismas foram analisados por meio de regressões lineares. Os modelos lineares de cada fc' não são estatisticamente significativos; entretanto, o modelo que considera todos os dados (ambos fc') apresenta um melhor ajuste.

Palavras-chave: Regressão linear; Transmissão indireta; Corpos prismáticos.

\section{INTRODUCCIÓN}


El hormigón es uno de los materiales de mayor demanda dentro de la industria de la construcción, debido a la facilidad de adquisición de sus materiales y simplicidad de ejecución (Silva et al., 2019). Sin embargo, en el proceso de elaboración queda expuesto a diversas condiciones que pueden afectar a sus propiedades físicas y mecánicas. Por esta razón, es necesario realizar un control de las características de los materiales empleados, dosificación de la mezcla, condiciones de curado, entre otros, para garantizar la resistencia proyectada (Kovler y Roussel, 2011; Wang y Subramaniam, 2011; Carcaño y Moreno, 2005). Mehta y Monteiro (2014) mencionan que la resistencia a la compresión del hormigón es influenciada por la relación $(\mathrm{a} / \mathrm{c})$, tamaño máximo del agregado, edad del hormigón y la velocidad de carga.

La determinación de la resistencia a la compresión del hormigón consiste en la aplicación de una velocidad de carga por unidad de área a un espécimen o muestra de hormigón (conocidos como cilindros) (ASTM, 2015). También puede ser determinada en estructuras existentes mediante la extracción de núcleos (ASTM, 2020); sin embargo, estos ensayos presentan limitaciones y pueden comprometer la estructura (Rocha y Póvoas, 2019). En este sentido, es importante determinar las características representativas del hormigón (resistencia a la compresión) sin dañar la estructura (International Atomic Energy Agency, 2000).

En la actualidad existen diversos ensayos no destructivos que permiten evaluar de forma rápida y eficaz el estado de una estructura, además de estimar sus propiedades mecánicas (Chang et al., 2003; McCann y Forde, 2001; Rehman et al., 2016).

De los diversos métodos no destructivos disponibles (McCann y Forde, 2001), la Velocidad de Pulso de Ultrasónico (VPU) ha demostrado varias aplicaciones en hormigón (Aggelis et al., 2010; Lorenzi et al., 2017; Silva et al., 2019), siendo empleada por más de 60 años para evaluar la calidad del hormigón (Kraub y Hariri, 2006). El ensayo se basa en la aplicación de la propagación de ondas ultrasónicas a través del hormigón, donde por medio de un transductor emisor y un receptor, la onda recorre el material; la velocidad se obtiene entre la división de la distancia de los transductores y el tiempo proporcionado por el equipo (Cánovas, 1988; Quispe, 2015). La medición de las velocidades puede ser realizada por tres métodos de operación: transmisión directa, indirecta y semidirecta, donde los transductores varían de posición (Mehta y Monteiro, 2014; IAEA, 2000; Malhotra y Carino, 2004; ASTM, 2003).

Cruz et al. (2014) señalan que la transmisión directa es la aplicación más deseable y satisfactoria al momento de evaluar la calidad del hormigón, puesto que los transductores se encuentran en las caras opuestas del material. Sin embargo, al realizar el ensayo in situ, no siempre se logra tener acceso a las caras opuestas de los elementos inspeccionados, por lo tanto, normalmente, se aplica la transmisión indirecta, realizando lecturas sobre una de las caras del elemento. En este sentido, la presente investigación tiene por objetivo estimar la resistencia a compresión de hormigón mediante el ensayo de VPU por transmisión indirecta.

\section{PROCEDIMIENTO}

Para alcanzar el objetivo de la presente investigación, se elaboraron cuerpos prismáticos de hormigón (20 cm x $20 \mathrm{~cm}$ x $25 \mathrm{~cm}$ ), donde se realizó el ensayo de VPU por transmisión indirecta. Posteriormente se efectuó una regresión lineal entre los resultados del ensayo de VPU y la resistencia a la compresión de los cuerpos prismáticos, valor determinado por medio de muestras cilíndricas. Se consideraron dos resistencias a la compresión de diseño (fc'), 21 y $28 \mathrm{MPa}$, siendo 6 cuerpos prismáticos cada una.

El procedimiento se realizó por medio de tres etapas. La primera consistió en la caracterización de los materiales para la elaboración del hormigón, además de definir la dosificación para las dos resistencias a la compresión de diseño ( $\left(\mathrm{fc}^{\prime}\right)$. En la segunda etapa se elaboraron los cuerpos prismáticos con sus respectivas muestras cilíndricas para la determinación de la resistencia a la 
compresión. Finalmente, en la tercera etapa, se efectuaron, tanto los ensayos de determinación de resistencia a la comprensión en las muestras cilíndricas como los de VPU en los cuerpos prismáticos (transmisión indirecta). Los datos fueron analizados mediante regresión lineal. La Figura 1 resume el procedimiento adoptado.

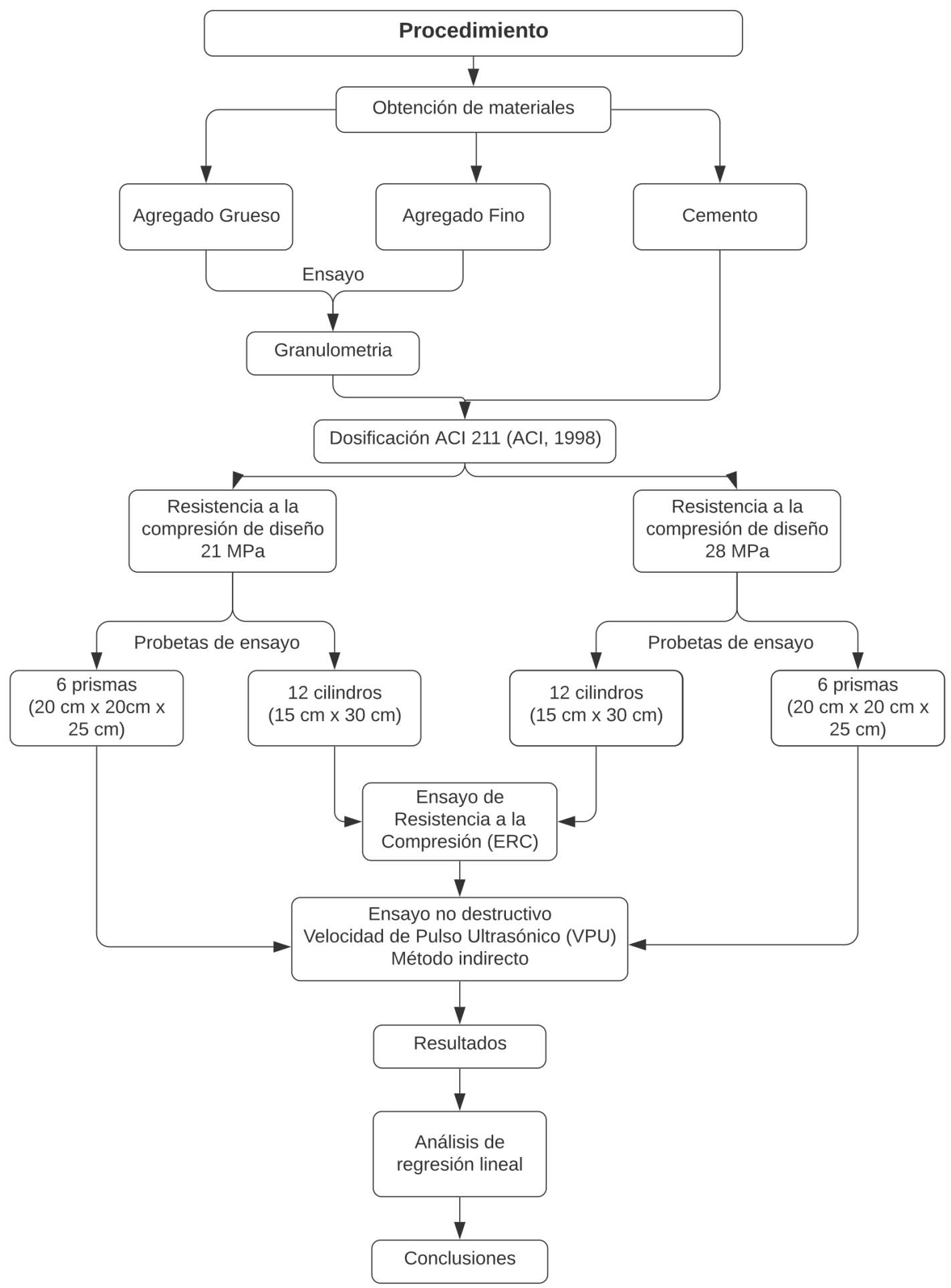

Figura 1. Procedimiento de la investigación

\subsection{Caracterización de los materiales utilizados}


El agregado grueso utilizado fue de tipo triturado proveniente de Tiquipaya, Cochabamba, Bolivia. El agregado fino utilizado fue de tipo rodado, extraído de Capinota, Cochabamba, Bolivia. El cemento utilizado fue IP-30 de la empresa COBOCE.

Se realizaron ensayos de granulometría siguiendo la normativa ASTM C136 (ASTM, 2006), para el agregado grueso y fino. Las Figuras 2 y 3, muestran los resultados obtenidos.

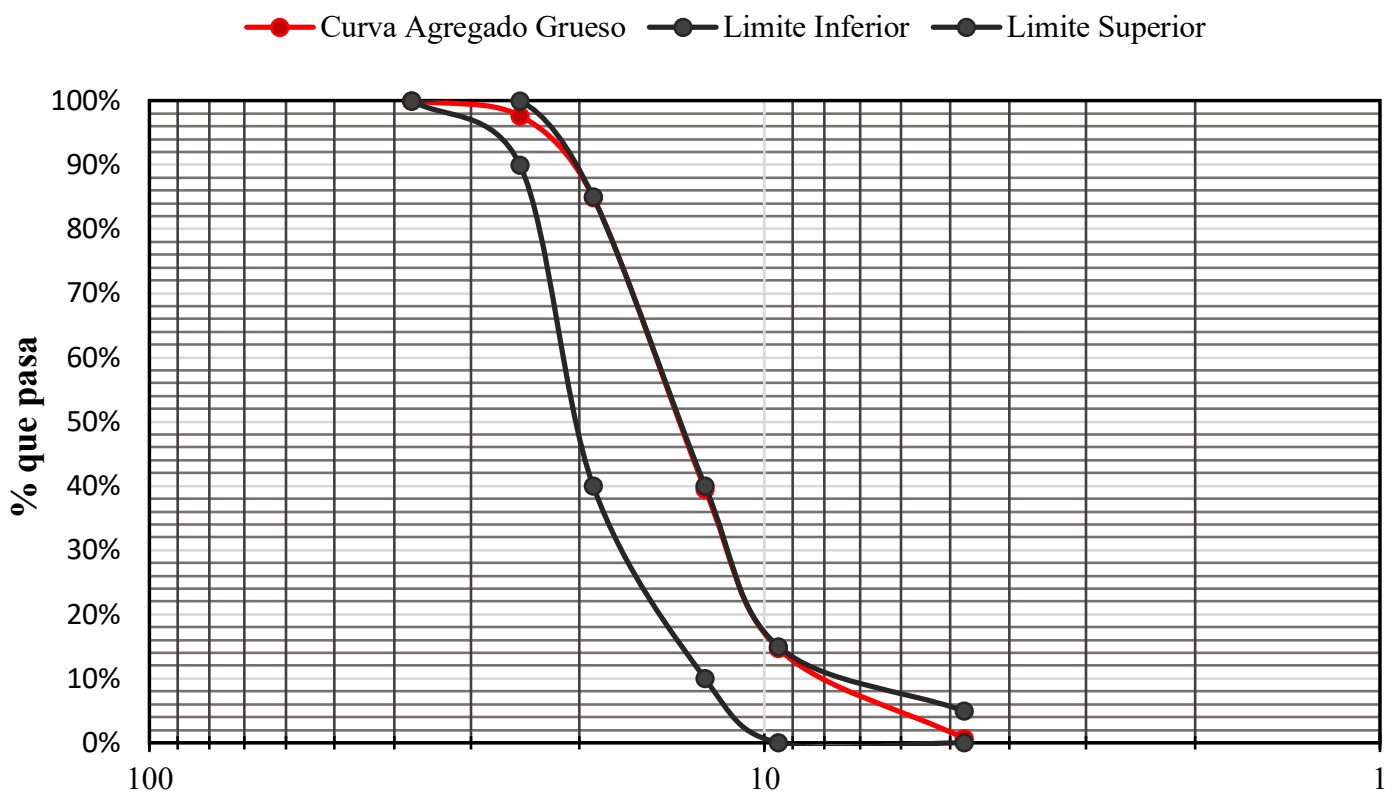

Abertura de los Tamices (mm)

Figura 2 - Curva granulométrica del agregado grueso

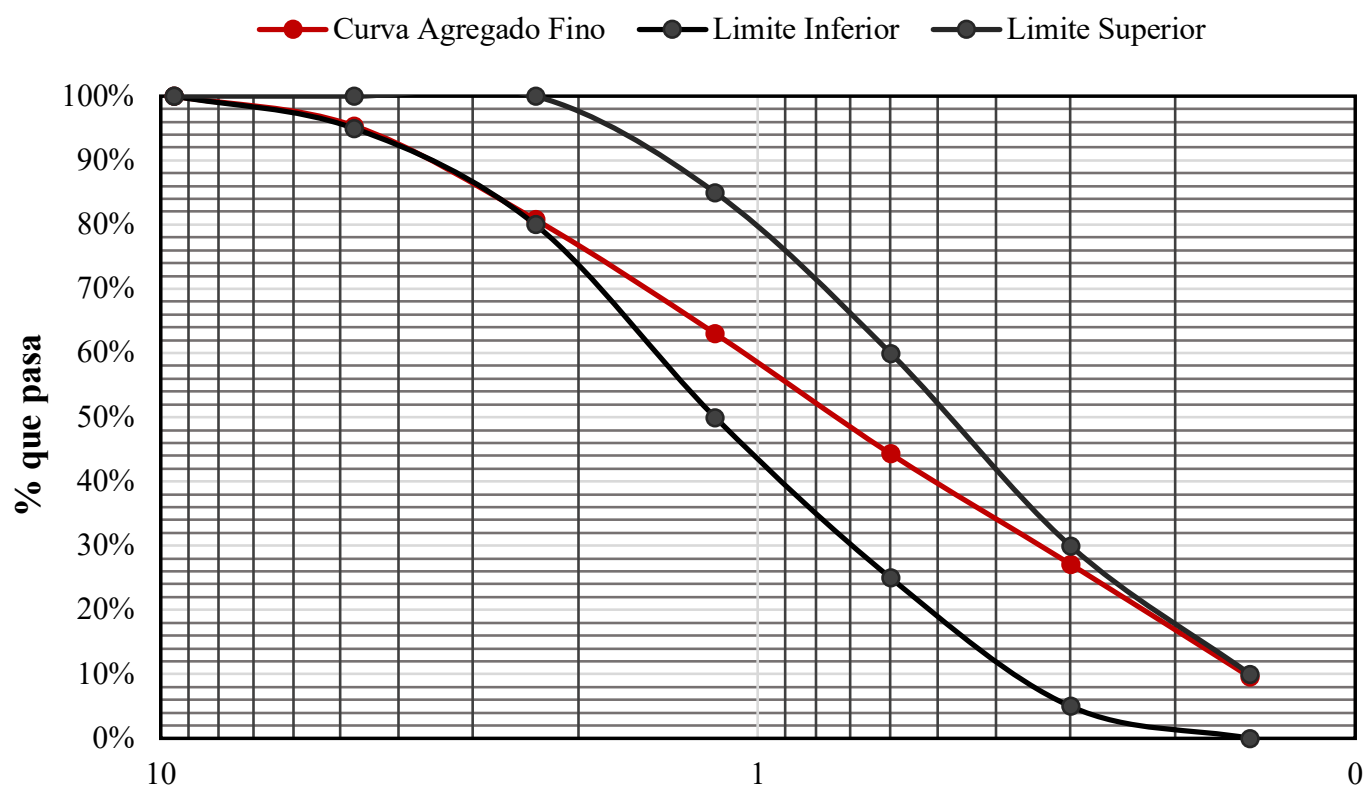

Abertura de los Tamices (mm)

Figura 3. Curva granulométrica del agregado fino 
Se realizaron dos dosificaciones de resistencia a la compresión siguiendo la norma ACI 318 (ACI, 2014). Para $21 \mathrm{MPa}$ se utilizó 1:2.24:5.48 (cemento:arena:grava) y una relación agua/cemento (a/c) de 0.64. En el caso de $28 \mathrm{MPa}, 1: 1.93: 4.96$ (cemento:arena:grava) y a/c de 0.58.

\subsection{Cuerpos prismáticos}

Fueron diseñados un total de 12 cuerpos prismáticos, 6 para cada resistencia a la compresión de diseño. La Figura 4 detalla las dimensiones de los cuerpos prismáticos.

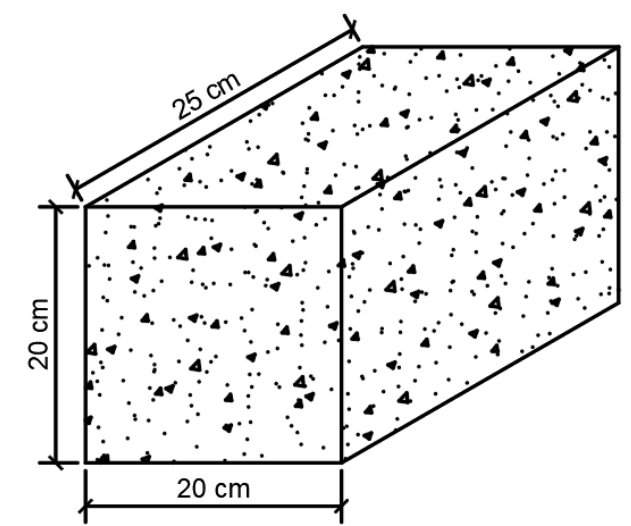

Figura 4. Dimensiones del cuerpo prismático

Durante su elaboración fueron vibrados mecánicamente a fin de eliminar los espacios vacíos y obtener un acabado liso. Se extrajeron dos muestras cilíndricas $(15 \mathrm{~cm}$ x $30 \mathrm{~cm})$ por cada cuerpo prismático, utilizando el promedio para la determinación de la resistencia a la compresión.

\subsection{Ensayos realizados}

a) Ensayo de resistencia a la compresión (RC)

A la edad de 28 días fueron ensayadas las muestras cilíndricas siguiendo la norma ASTM C39 (ASTM, 2015). Previamente se tomaron las medidas y pesos de las probetas cilíndricas. La Figura 5 detalla la realización del ensayo.
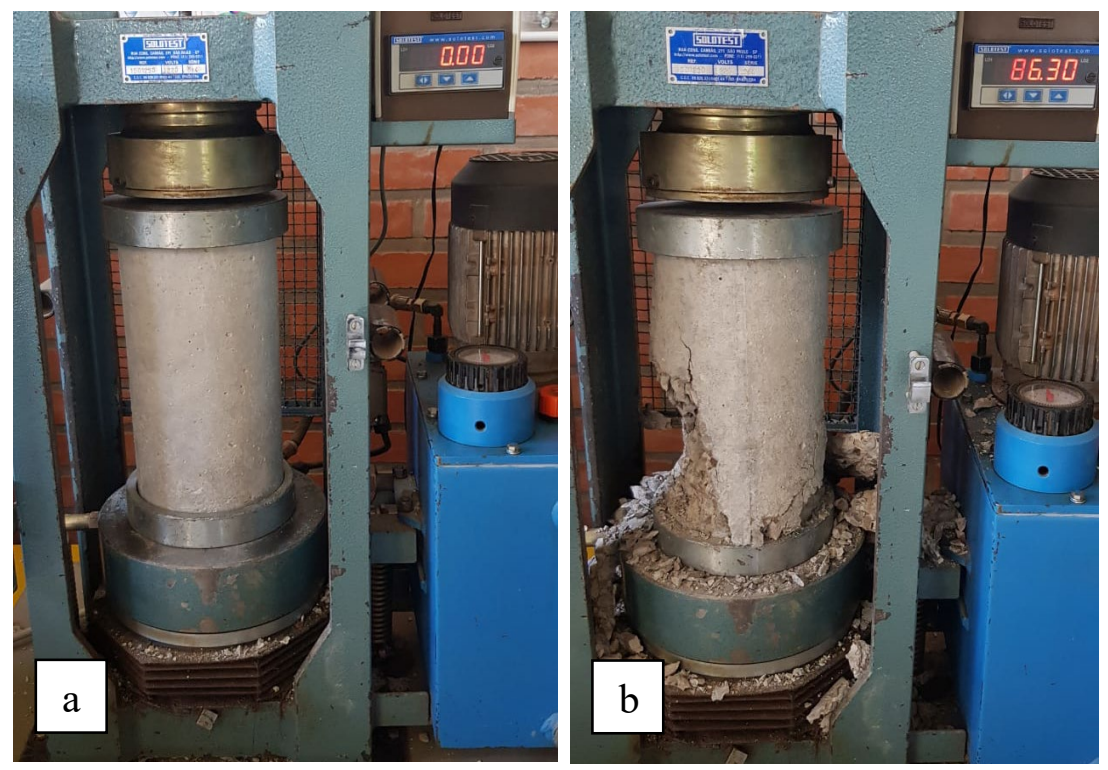

Figura 5. Ensayo de resistencia a la compresión: a) sin carga y b) con carga 


\section{b) Ensayo Velocidad de Pulso Ultrasónico (VPU)}

A la misma edad de la determinación de la resistencia a la compresión, 28 días, se realizó el ensayo de VPU por transmisión indirecta en los cuerpos prismáticos. Para esto se marcaron dos líneas sobre la cara a ser ensayada, como se muestra en la siguiente Figura 6.

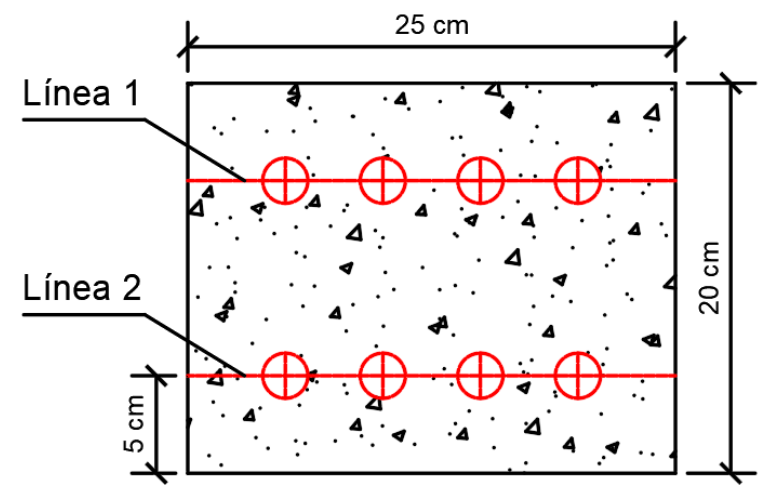

Figura 6. Esquema de la cara ensayada

El transductor emisor se mantuvo en una posición fija y el transductor receptor fue cambiando de posición cada $5 \mathrm{~cm}$, teniendo en total tres mediciones por línea como se muestra en la Figura 7.
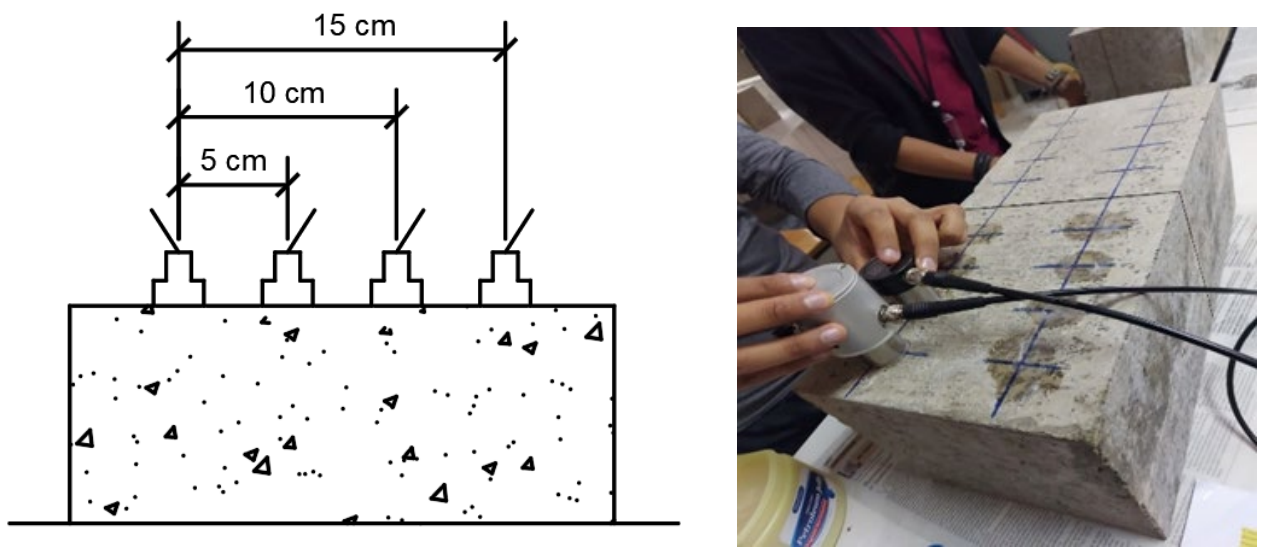

Figura 7. Ensayo de VPU - transmisión indirecta

\subsection{Análisis de datos}

Los datos fueron analizados mediante regresión lineal utilizando el programa Minitab 19. En total se realizaron tres regresiones lineales: $\mathrm{A}$, para los cuerpos prismáticos $\mathrm{fc}^{\prime}=21 \mathrm{MPa}$; $\mathrm{B}$, para los cuerpos prismáticos $\mathrm{fc}^{\prime}=28 \mathrm{MPa}, \mathrm{y} \mathrm{C}$, para todos los cuerpos prismáticos (ambos fc').

\section{RESULTADOS}

En las Tablas 1 y 2 se presentan los resultados de los ensayos de VPU y RC para fc' de 21 y 28 $\mathrm{MPa}$, respectivamente.

Para el ensayo de VPU se sacó el promedio de tiempo (Líneas 1 y 2) por cada distancia $(5 \mathrm{~cm}, 10$ $\mathrm{cm}$ y $15 \mathrm{~cm}$ ), posteriormente se calculó la velocidad de cada medición en m/s. Finalmente se obtuvo la media de las tres velocidades por cuerpo prismático, este valor fue utilizado para la regresión lineal con la resistencia a la compresión de las muestras cilíndricas. 
Tabla 1. Resultados de VPU y RC para $\mathrm{fc}^{\prime}=21 \mathrm{MPa}$

\begin{tabular}{|c|c|c|c|c|c|c|c|}
\hline \multirow{2}{*}{$\begin{array}{c}\text { Cuerpo } \\
\text { prismático }\end{array}$} & \multirow{2}{*}{$\begin{array}{l}\text { Distancia } \\
\quad(\mathrm{cm})\end{array}$} & \multicolumn{2}{|c|}{ Tiempo ( $\mu \mathrm{s})$} & \multirow{2}{*}{$\begin{array}{c}\text { Media del } \\
\text { tiempo } \\
(\mu \mathrm{s}) \\
\end{array}$} & \multirow{2}{*}{ VPU (m/s) } & \multirow{2}{*}{$\begin{array}{c}\text { VPU } \\
\text { promedio } \\
(\mathrm{m} / \mathbf{s})\end{array}$} & \multirow{2}{*}{$\begin{array}{c}\text { RC } \\
\text { promedio } \\
\text { (MPa) }\end{array}$} \\
\hline & & Línea 1 & Línea 2 & & & & \\
\hline \multirow{3}{*}{$\mathrm{CP} 1$} & 5 & 19.30 & 18.60 & 18.95 & 2638.52 & \multirow{3}{*}{2720.32} & \multirow{3}{*}{20.24} \\
\hline & 10 & 38.10 & 33.20 & 35.65 & 2805.05 & & \\
\hline & 15 & 63.80 & 46.60 & 55.20 & 2717.39 & & \\
\hline \multirow{3}{*}{$\mathrm{CP} 2$} & 5 & 19.90 & 15.50 & 17.70 & 2824.86 & \multirow{3}{*}{2957.50} & \multirow{3}{*}{25.90} \\
\hline & 10 & 33.00 & 33.90 & 33.45 & 2989.54 & & \\
\hline & 15 & 46.60 & 51.50 & 49.05 & 3058.10 & & \\
\hline \multirow{3}{*}{$\mathrm{CP} 3$} & 5 & 15.10 & 19.30 & 17.20 & 2906.98 & \multirow{3}{*}{2727.30} & \multirow{3}{*}{20.42} \\
\hline & 10 & 33.00 & 45.20 & 39.10 & 2557.54 & & \\
\hline & 15 & 56.40 & 54.00 & 55.20 & 2717.39 & & \\
\hline \multirow{3}{*}{$\mathrm{CP} 4$} & 5 & 17.50 & 16.30 & 16.90 & 2958.58 & \multirow{3}{*}{2920.68} & \multirow{3}{*}{23.28} \\
\hline & 10 & 36.40 & 41.40 & 38.90 & 2570.69 & & \\
\hline & 15 & 42.70 & 50.10 & 46.40 & 3232.76 & & \\
\hline \multirow{3}{*}{ CP5 } & 5 & 17.30 & 15.40 & 16.35 & 3058.10 & \multirow{3}{*}{2879.89} & \multirow{3}{*}{20.89} \\
\hline & 10 & 44.70 & 30.80 & 37.75 & 2649.01 & & \\
\hline & 15 & 56.90 & 45.40 & 51.15 & 2932.55 & & \\
\hline \multirow{3}{*}{ CP6 } & 5 & 17.80 & 18.60 & 18.20 & 2747.25 & \multirow{3}{*}{2929.06} & \multirow{3}{*}{23.43} \\
\hline & 10 & 31.50 & 31.50 & 31.50 & 3174.60 & & \\
\hline & 15 & 50.60 & 54.10 & 52.35 & 2865.33 & & \\
\hline
\end{tabular}

Tabla 2. Resultados de VPU y RC para $\mathrm{fc}^{\prime}=28 \mathrm{MPa}$

\begin{tabular}{|c|c|c|c|c|c|c|c|}
\hline \multirow{2}{*}{$\begin{array}{c}\text { Cuerpo } \\
\text { prismático }\end{array}$} & \multirow{2}{*}{$\begin{array}{l}\text { Distancia } \\
\text { (cm) }\end{array}$} & \multicolumn{2}{|c|}{ Tiempo ( $\mu \mathrm{s})$} & \multirow{2}{*}{$\begin{array}{c}\text { Media del } \\
\text { tiempo } \\
(\mu \mathrm{s})\end{array}$} & \multirow{2}{*}{ VPU (m/s) } & \multirow{2}{*}{$\begin{array}{c}\text { VPU } \\
\text { promedio } \\
(\mathbf{m} / \mathbf{s})\end{array}$} & \multirow{2}{*}{$\begin{array}{c}\mathrm{RC} \\
\text { promedio } \\
\text { (MPa) }\end{array}$} \\
\hline & & Línea 1 & Línea 2 & & & & \\
\hline \multirow{3}{*}{$\mathrm{CP} 7$} & 5 & 15.70 & 13.00 & 14.35 & 3484.32 & \multirow{3}{*}{3561.68} & \multirow{3}{*}{35.29} \\
\hline & 10 & 26.70 & 28.80 & 27.75 & 3603.60 & & \\
\hline & 15 & 40.50 & 42.90 & 41.70 & 3597.12 & & \\
\hline \multirow{3}{*}{ CP8 } & 5 & 17.30 & 16.40 & 16.85 & 2967.36 & \multirow{3}{*}{3180.75} & \multirow{3}{*}{29.81} \\
\hline & 10 & 29.80 & 32.40 & 31.10 & 3215.43 & & \\
\hline & 15 & 46.10 & 43.20 & 44.65 & 3359.46 & & \\
\hline \multirow{3}{*}{ CP9 } & 5 & 18.10 & 18.30 & 18.20 & 2747.25 & \multirow{3}{*}{3045.11} & \multirow{3}{*}{27.94} \\
\hline & 10 & 31.20 & 31.50 & 31.35 & 3189.79 & & \\
\hline & 15 & 50.10 & 43.70 & 46.90 & 3198.29 & & \\
\hline \multirow{3}{*}{ CP10 } & 5 & 17.10 & 15.30 & 16.20 & 3086.42 & \multirow{3}{*}{3255.67} & \multirow{3}{*}{29.54} \\
\hline & 10 & 32.00 & 29.50 & 30.75 & 3252.03 & & \\
\hline & 15 & 42.70 & 44.80 & 43.75 & 3428.57 & & \\
\hline \multirow{3}{*}{$\mathrm{CP} 11$} & 5 & 15.90 & 17.00 & 16.45 & 3039.51 & \multirow{3}{*}{3226.34} & \multirow{3}{*}{28.63} \\
\hline & 10 & 29.40 & 32.00 & 30.70 & 3257.33 & & \\
\hline & 15 & 41.30 & 47.40 & 44.35 & 3382.19 & & \\
\hline \multirow{3}{*}{ CP12 } & 5 & 13.30 & 16.30 & 14.80 & 3378.38 & \multirow{3}{*}{3477.94} & \multirow{3}{*}{30.56} \\
\hline & 10 & 28.60 & 30.20 & 29.40 & 3401.36 & & \\
\hline & 15 & 41.30 & 40.80 & 41.05 & 3654.08 & & \\
\hline
\end{tabular}


En las Figuras 8 y 9 se presenta la regresión lineal para los dos tipos de hormigón elaborados junto con los resultados del análisis de varianza (Tabla 3 y 4 ).

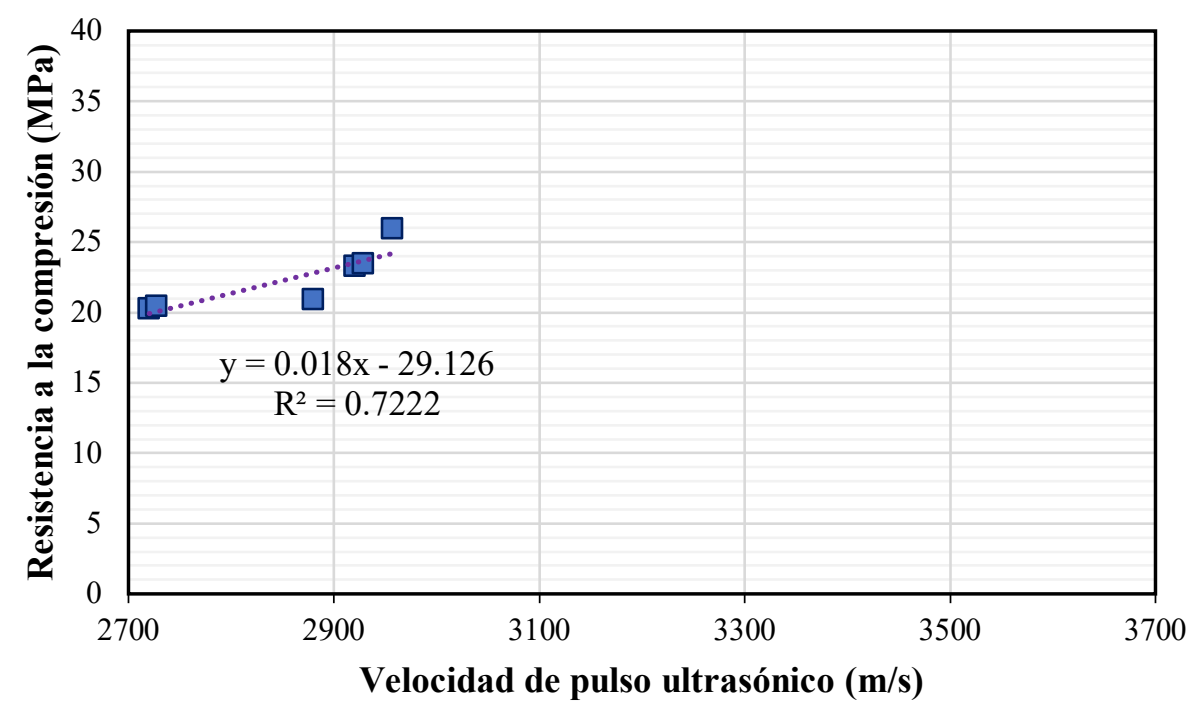

Figura 8. Regresión lineal A

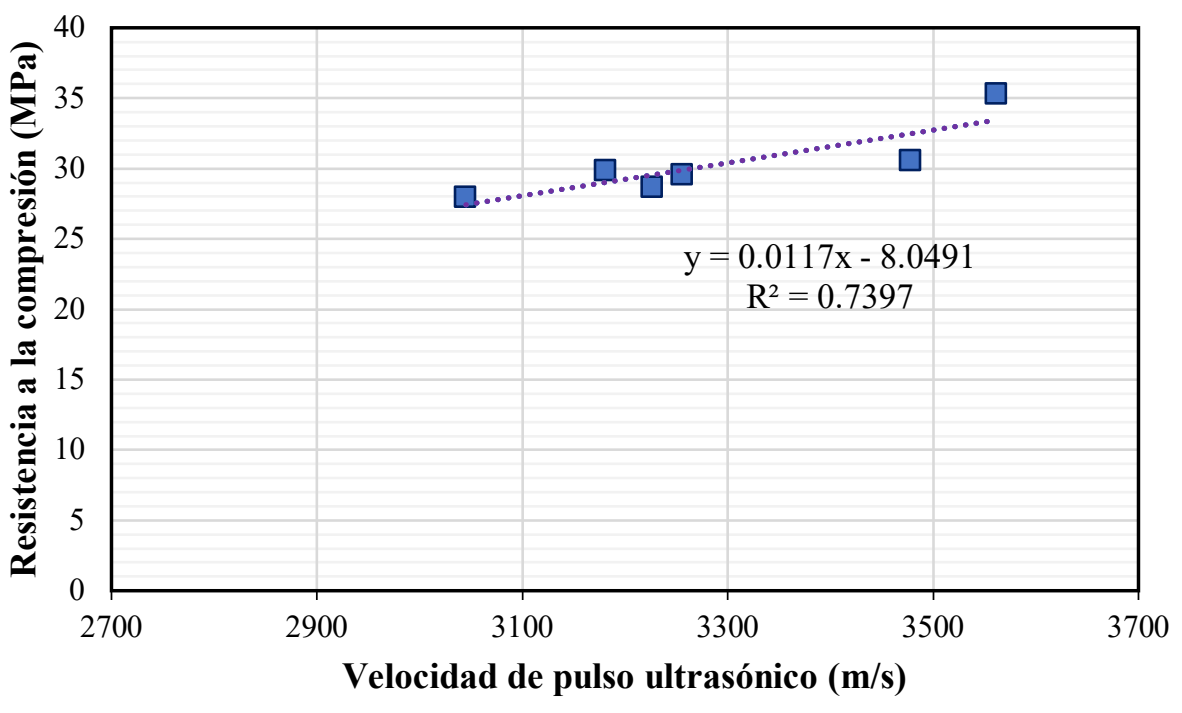

Figura 9. Regresión lineal B

Tabla 3. Análisis de varianza para la regresión lineal A

\begin{tabular}{|c|c|c|c|c|c|}
\hline Fuente & $\begin{array}{c}\text { Grado } \\
\text { de } \\
\text { libertad }\end{array}$ & $\begin{array}{c}\text { Suma de } \\
\text { cuadrados }\end{array}$ & $\begin{array}{c}\text { Cuadrados } \\
\text { medios }\end{array}$ & $\begin{array}{c}\text { Valor } \\
\text { F }\end{array}$ & $\begin{array}{c}\text { Valor } \\
\text { P }\end{array}$ \\
\hline Regresión & 1 & 17.99 & 17.99 & 10.40 & 0.032 \\
\hline VPU & 1 & 17.99 & 17.99 & 10.40 & 0.032 \\
\hline Error & 4 & 6.92 & 1.73 & & \\
\hline Total & 5 & 24.92 & & & \\
\hline
\end{tabular}


Tabla 4. Análisis de varianza para la regresión lineal B

\begin{tabular}{|c|c|c|c|c|c|}
\hline Fuente & $\begin{array}{c}\text { Grado } \\
\text { de } \\
\text { libertad }\end{array}$ & $\begin{array}{c}\text { Suma de } \\
\text { los } \\
\text { cuadrados }\end{array}$ & $\begin{array}{c}\text { Cuadrados } \\
\text { medios }\end{array}$ & $\begin{array}{c}\text { Valor } \\
\text { F }\end{array}$ & $\begin{array}{c}\text { Valor } \\
\text { P }\end{array}$ \\
\hline Regresión & 1 & 25.28 & 25.28 & 11.37 & 0.028 \\
\hline VPU & 1 & 25.28 & 25.28 & 11.37 & 0.028 \\
\hline Error & 4 & 8.90 & 2.22 & & \\
\hline Total & 5 & 34.18 & & & \\
\hline
\end{tabular}

Se observa que el coeficiente de determinación $\left(\mathrm{R}^{2}\right)$ es medio para ambos casos, 0.7222 y 0.7397 , siendo más cercano a 0.50 que a 1 . Esto indica que los valores estimados de la regresión lineal no son cercanos a los valores reales.

Para el análisis de varianza se utilizó el estadístico F (Tablas 3 y 4), el cual compara el ajuste del modelo lineal con un modelo sin predictores (modelo de solo intercepción). Para esto se plantean dos hipótesis: Hipótesis nula, H0: El modelo lineal y de solo intercepción son iguales, e Hipótesis Alterna, H1: el ajuste del modelo de solo intercepción se reduce significativamente en comparación del modelo lineal. Para la presente investigación se utilizó un nivel de significancia de 0.01 .

Para ambos modelos el valor $\mathrm{P}$ del estadístico $\mathrm{F}$ es mayor que el nivel de significancia, por tanto, se acepta la $\mathrm{H} 0$, los modelos lineales propuestos y de solo intercepción son iguales. Esto quiere decir que las regresiones realizadas no son estadísticamente significativas.

Sin embargo, cuando se consideran todos los datos para la regresión lineal, se observa que el $\mathrm{R}^{2}$ es elevado, 0.9114 (Figura 10). El modelo propuesto se ajusta mejor a los datos.

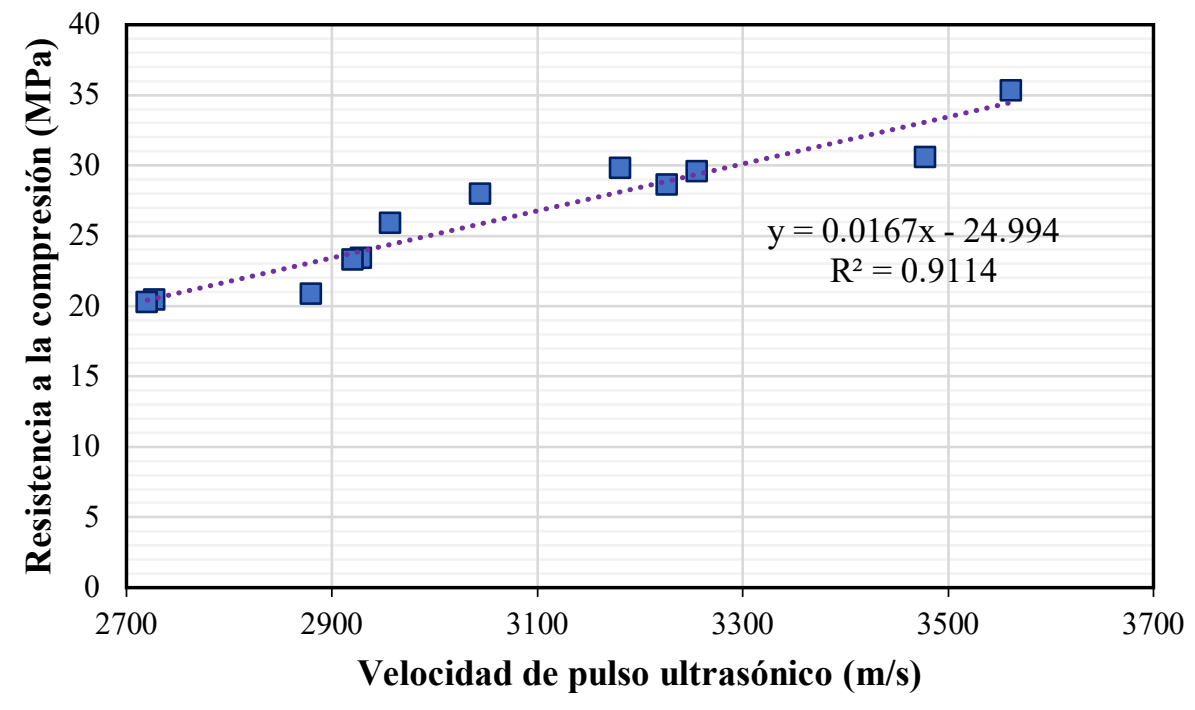

Figura 10. Regresión lineal C

La Tabla 5 presenta el análisis de varianza para la regresión lineal C, donde el valor P del estadístico $\mathrm{F}$ es menor que el nivel de significancia, se acepta la H1. Entonces el modelo de regresión es estadísticamente significativo, puede ser utilizado para estimar la resistencia a la compresión del hormigón a partir de ensayos de VPU; sin embargo, el modelo se limita a las velocidades de 2700 a $3700 \mathrm{~m} / \mathrm{s}$, aproximadamente.

Adicionalmente, para determinar la significancia del coeficiente de correlación de Pearson $(R)$ se aplicó la prueba t-student. Se plantearon dos hipótesis: Hipótesis nula, H0: $\mathrm{R}=0$, no existe 
correlación lineal, e Hipótesis alterna, $\mathrm{H} 1: \mathrm{R} \neq 0$, existe correlación lineal. Para esto se calcula el valor $t$ con la ecuación (1), donde $N$ es el número de datos, 12.

Tabla 5. Análisis de varianza para la regresión lineal C

\begin{tabular}{|c|c|c|c|c|c|}
\hline Fuente & $\begin{array}{c}\text { Grado } \\
\text { de } \\
\text { libertad }\end{array}$ & $\begin{array}{c}\text { Suma de } \\
\text { cuadrados }\end{array}$ & $\begin{array}{c}\text { Cuadrados } \\
\text { medios }\end{array}$ & $\begin{array}{c}\text { Valor } \\
\text { F }\end{array}$ & $\begin{array}{c}\text { Valor } \\
\mathbf{P}\end{array}$ \\
\hline Regresión & 1 & 225.99 & 225.99 & 102.81 & 0.000 \\
\hline VPU & 1 & 225.99 & 225.99 & 102.81 & 0.000 \\
\hline Error & 10 & 21.98 & 2.198 & & \\
\hline Total & 11 & 247.98 & & & \\
\hline \multicolumn{5}{c}{$t=\frac{R}{\sqrt{\frac{1-R^{2}}{N-2}}} \quad(1)$} \\
\end{tabular}

El valor $t$ fue 10.41. El $t_{\alpha, \mathrm{N}-2}$ fue obtenido de las tablas de distribución $t$-student, 3.169, para un $\alpha$ (nivel de significancia) de 0.01 . Donde $t>t_{\alpha, \mathrm{N}-2}$, se acepta la hipótesis alterna, existe correlación lineal.

De los modelos lineales presentados, se observa que los valores de VPU incrementan con la resistencia a compresión del hormigón. El valor del $\mathrm{R}^{2}$ es elevado, en especial para la regresión lineal C, resultados también reportados por Silva et al. (2019) y Güneyli et al. (2017). Alcañiz et al. (2015) confirman que existe una correlación positiva y estadísticamente significativa entre la resistencia a la compresión del hormigón y los valores de VPU; sin embargo, gran parte de los estudios sobre el asunto solo consideran datos de VPU por transmisión directa. La presente investigación demuestra que la utilización datos de VPU por transmisión indirecta proporciona, de igual forma, resultados positivos y significativos.

Si bien existen muchos estudios de regresión lineal entre la resistencia a la compresión y VPU, otras investigaciones han presentado relaciones exponenciales con igual o mejores resultados (Tharmaratnam y Tan, 1990). Incluso la utilización de redes neuronales puede estimar la resistencia a compresión con errores menores al 5\% (Silva et al., 2021)

\section{CONCLUSIONES}

En la presente investigación se propuso un modelo de regresión lineal entre los datos obtenidos por el ensayo VPU (transmisión indirecta) y de resistencia a la compresión. Se demostró que los modelos lineales para cada fc', no presentan un ajuste de regresión lineal estadísticamente significativo. Mientras que, si se consideran todos los datos (ambos fc'), el modelo presenta mejor ajuste. A mayor cantidad de datos y consideración de otras resistencias a la compresión de diseño, el modelo adquiere mayor significancia.

La tendencia de la regresión es positiva, indicando que a mayor VPU, la resistencia a la compresión aumenta, esto debido a que la onda ultrasónica viaja de forma más rápida en hormigones de mayor calidad (menores espacios vacíos y menor relación a/c), como es reportado en la literatura.

Si bien el modelo propuesto presenta un buen ajuste, está limitado a las características de los materiales y condiciones del ensayo. Investigaciones futuras pueden considerar otras variables y observar su influencia en la estimación de la resistencia a la compresión del hormigón por medio de ensayos de VPU, tales como distancia entre los transductores y transmisión semidirecta. Incluso se pueden considerar modelos que no sean necesariamente lineales para obtener una mejor estimación de la resistencia a la compresión. 


\section{REFERENCIAS}

Aggelis, D. G., Kordatos, E. Z., Soulioti, D. V., and Matikas, T. E. (2010), Combined use of thermography and ultrasound for the characterization of subsurface cracks in concrete. Construction and Building Materials. 24(10):1888-1897. https://doi.org/10.1016/j.conbuildmat.2010.04.014

Alcañiz, J., Louis, M., Sánchez, F. and Lasheras, A. (2015), Estudio en estructuras de hormigón armado. Velocidad de ultrasonidos y resistencia a compresión. Revista Científica, 21:19-28. https://doi.org/10.14483/udistrital.jour.RC.2015.21.a2

American Concrete Institute - ACI. (2014), ACI CODE-318-14: Building Code Requirements for Structural Concrete and Commentary. ACI, Estados Unidos.

American Society for Standard Testing and Materials - ASTM. (2015), ASTM C39 Standard Test Method for Compressive Strength of Cylindrical Concrete Specimens. https://doi.org/10.1520/C0039

American Society of Testing and Materials - ASTM. (2003), ASTM C597-03 Standard Test method for pulse velocity through concrete. https://doi.org/10.1520/C0597-16

American Society of Testing and Materials - ASTM. (2006), ASTM C136-06 Standard test method for sieve analysis of fine and coarse aggregates. https://doi.org/10.1520/C0136-06

American Society of Testing and Materials - ASTM. (2020), ASTM C42/C42M-20 Standard Test

Method for Obtaining and Testing Drilled Cores and Sawed Beams of Concrete. https://doi.org/10.1520/C0042 C0042M-20

International Atomic Energy Agency - IAEA. Guidebook on non-destructive testing of concrete structures. IAEA, Austria, 2000, 100-128.

Cánovas, M.F. (1988), “Patologia e terapia do concreto armado”. PINI, São Paulo, Brasil, p. 522. Carcaño, S. and Moreno, E. (2005), Influencia del curado húmedo en la resistencia a compresión del concreto en clima cálido sub-húmedo. Ingeniería Revista Académica. 9(3):5-17.

Chang, P., Flatau, A., and Liu, S. (2003), Review paper: health monitoring of civil infrastructure. Structural Health Monitoring. 2(3):257-267.

Cruz, R., Quintero, L. A., and Herrera, J. (2014), Evaluación del efecto de barras de refuerzo en concreto sobre las medidas de velocidad de pulso ultrasónico (VPU). Revista Colombiana de Materiales. (5):107-113.

Güneyli, H., Karahan, S. and Güneyli, A. (2017), Water content and temperature effect on ultrasonic pulse velocity of concrete. Russian Journal of Nondestructive Testing, 53(2):159-166. https://doi.org/10.1134/S1061830917020024

International Atomic Energy Agency. (2002). “Guidebook on non-destructive testing of concrete structures". IAEA, Viena, Austria, p. 242.

Kovler, K. and Roussel, N. (2011), Properties of Fresh and Hardened Concrete. Cement and Concrete Research. 41(7):775-792. https://doi.org/10.1016/j.cemconres.2011.03.009

Kraub, M. and Hariri, K. (2006), Determination of initial degree of hydration for improvement of early-age properties of concrete using ultrasonic wave propagation. Cement and Concrete Composites. 28(4):299-306. https://doi.org/10.1016/j.cemconcomp.2006.02.007

Lorenzi, A., Argenta Chies, J., Santos Adamatti, D., and Pinto da Silva Filho, L. C. (2017), Evaluation of concrete flaw detection capability by means of ultrasonic tests. Revista ALCONPAT. 7(3):286-301. https://doi.org/10.21041/ra.v7i3.127

Malhotra, V. M., Carino, N. J. (2004), “Handbook on nondestructive testing of concrete”. ASTM International CRC Press, United States of America, p. 384. 
McCann, D. M., and Forde, M. C. (2001), Review of NDT methods in the assessment of concrete and masonry structures. Ndt \& E International. 34(2):71-84. https://doi.org/10.1016/S0963$\underline{8695(00) 00032-3}$

Mehta, P. K., Monteiro P. J. (2014), “Concrete, Microstructure, properties, and materials”. Mc Graw Hill, United States of America, p. 704.

Quispe, L. E. A. (2015), Resistencia a la compresión del concreto a partir de la velocidad de pulsos de ultrasonido. Civilizate. (6):18-20.

Rehman, S. K. U., Ibrahim, Z., Memon, S. A. and Jameel, M. (2016), Nondestructive test methods for concrete bridges. Construction and building materials. 107:58-86. http://dx.doi.org/10.1016/j.conbuildmat.2015.12.011

Rocha, J. H. A., and Póvoas, Y. V. (2019), Detection of Delaminations in Reinforced Concrete Bridges Using Infrared Thermography. Revista ingeniería de construcción. 34(1):55-64. https://dx.doi.org/10.4067/S0718-50732019000100055

Silva, M. T., Rocha, J. H. A. and Monteiro, E. C. B. (2019), Estimação da profundidade de fissuras em concreto através da velocidade de ondas ultrassônicas. Matéria (Rio de Janeiro). 24(4). https://doi.org/10.1590/S1517-707620190004.0847

Silva, F. A. N., Nogueira, C. L., Silva, J. A., Araújo, A. V. P., Azevedo, A. C. and Delgado, J. M. P. Q. (2019), Ultrasonic Assessment of Damage in Concrete under Compressive and Thermal Loading Using Longitudinal and Transverse Waves. Russian Journal of Nondestructive Testing, 55(11):808-816. https://doi.org/10.1134/s1061830919110081

Silva, F. A., Delgado, J. M., Cavalcanti, R. S., Azevedo, A. C., Guimarães, A. S. and Lima, A. G. (2021), Use of Nondestructive Testing of Ultrasound and Artificial Neural Networks to Estimate Compressive Strength of Concrete. Buildings, $11(2): 44$. https://doi.org/10.3390/buildings11020044

Tharmaratnam, K., and Tan, B. S. (1990), Attenuation of ultrasonic pulse in cement mortar. Cement and Concrete research, 20(3):335-345. https://doi.org/10.1016/0008-8846(90)90022-P

Wang, X. and Subramaniam, K. V. (2011), Ultrasonic Monitoring of Capillary Porosity and Elastic Properties in Hydrating Cement Paste. Cemente \& Concrete Composites. 33(3):389-401. https://doi.org/10.1016/j.cemconcomp.2010.11.001 